\title{
ESTUDO ELETROQUÍMICO E QUÍMICO-QUÂNTICO DA OXIDAÇÃO DO ANTIDEPRESSIVO TRICÍCLICO AMITRIPTILINA
}

\author{
Renata Alves de Toledo e Luiz Henrique Mazo* \\ Departamento de Físico-Química, Instituto de Química de São Carlos, Universidade de São Paulo, CP 780, \\ 13560-970 São Carlos - SP \\ Mauro Coelho dos Santos \\ Departamento de Química, Universidade Federal de São Carlos, CP 676, 13560-970 São Carlos - SP \\ Káthia Maria Honório, Albérico Borges Ferreira da Silva e Éder Tadeu Gomes Cavalheiro \\ Departamento de Química e Física Molecular, Instituto de Química de São Carlos, Universidade de São Paulo, CP 780, \\ 13560-970 São Carlos - SP
}

Recebido em 29/4/04; aceito em 25/10/04; publicado na web em 4/2/05

\begin{abstract}
ELECTROCHEMICAL AND QUANTUM-CHEMICAL STUDIES OF THE OXIDATION OF THE ANTIDEPRESSANT AMITRIPTYLINE. This work presents the electrochemical and quantum chemical studies of the oxidation of the tricyclic antidepressant amitriptyline (AM) employing a carbon-polyurethane composite electrode (GPU) in a $0.1 \mathrm{~mol} \mathrm{~L}^{-1} \mathrm{BR}$ buffer. The electrochemical results showed that the oxidation of AM occurs irreversibly at potentials close to $830 \mathrm{mV}$ with the loss of one electron and one proton and is controlled by reagent and product adsorption. According to the PM3 results, the atom C16 is the region of highest probability for the oxidation of AM since it has the largest charge variation.
\end{abstract}

Keywords: amitriptyline; composite electrode; PM3 semi-empirical method.

\section{INTRODUÇÃO}

A depressão é um transtorno afetivo que é caracterizada por uma alteração psíquica e orgânica com conseqüentes modificações na maneira de avaliar a realidade e a vida. Os antidepressivos são medicamentos que ajudam a restaurar o equilíbrio químico no cérebro, aumentando a concentração de norepinefrina ou de serotonina na sinapse no sistema nervoso central ${ }^{1}$.

A amitriptilina, 3-(10,11)-di-hidro-5H-dibenzo[a,d] ciclo-hepteno-5-ilideno)-N,N-dimentil-1-propanamina, cuja estrutura é mostrada na Figura 1, é um antidepressivo da classe dos tricíclicos, muito utilizado no tratamento da síndrome depressiva e de estados de ansiedade associados com depressão ${ }^{2}$

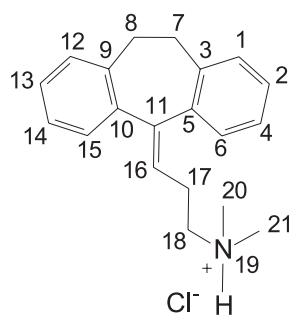

Figura 1. Estrutura da amitriptilina

A escassez de trabalhos na literatura que abordem o comportamento voltamétrico e, conseqüentemente, alguns aspectos mecanísticos está relacionada com a pequena eletroatividade da amitriptilina sobre superfícies eletródicas convencionais, tais como o ouro e a platina $^{3}$. Deste modo, o desenvolvimento de eletrodos quimicamente

*e-mail: 1hmazo@iqsc.usp.br modificados faz-se necessário a fim de permitir o estudo de suas reações de oxidação e, também, auxiliar na elaboração de novas metodologias analíticas, que permitam a determinação de traços do composto em amostras biológicas.

A detecção eletroquímica de alguns antidepressivos tricíclicos, dentre eles a amitriptilina, foi estudada sobre eletrodo de carbono vítreo reticulado contendo eletrodepósitos de policarbazol e politiofeno ${ }^{4}$. O perfil voltamétrico da amitriptilina obtido com a modificação polimérica deve-se à presença de átomos doadores de elétrons, tais como enxofre (politiofeno) e nitrogênio (policarbazol), que facilitam a formação do radical cátion.

A modificação do eletrodo de pasta de carbono com poli(Nvinilimidazol) para a determinação voltamétrica de amitriptilina foi relatada por Biryol e colaboradores ${ }^{5}$. Neste trabalho, a adição do modificador na pasta de carbono foi responsável pelo aumento na sensibilidade analítica e maior definição do perfil do pico de oxidação, permitindo assim o desenvolvimento de metodologia analítica para a determinação de amitriptilina em formulações farmacêuticas.

Recentemente, a larga janela eletroquímica, a alta estabilidade em meios agressivos, a reprodutibilidade e a sensibilidade do eletrodo de diamante dopado com boro têm permitido a detecção de antidepressivos tricíclicos, dentre eles a amitriptilina, em amostras de sangue com limite de detecção de $16,3 \times 10^{-8} \mathrm{~mol} \mathrm{~L}^{-1}{ }^{6}$.

Um aumento expressivo no número de trabalhos na literatura utilizando eletrodos quimicamente modificados, nas mais variadas matrizes, está intimamente relacionado com a melhora na seletividade, sensibilidade, estabilidade e tempo de vida do eletrodo ${ }^{7}$. O eletrodo compósito grafite/poliuretana (GPU), preparado de acordo a metodologia de Mendes e colaboradores ${ }^{8}$, vem agrupando tais características, o que resulta, juntamente com o emprego de técnicas voltamétricas de pulso, em uma diminuição do limite de detecção, atingindo níveis comparáveis aos das técnicas cromatográficas.

As técnicas voltamétricas permitem extrair vários parâmetros 
importantes para o estudo do comportamento oxidativo de biomoléculas, dentre eles o grau da reversibilidade da reação, a ocorrência ou não de processos adsortivos na superfície do eletrodo e o número de prótons e elétrons envolvidos na reação. No entanto, uma ferramenta que vem sendo bastante utilizada para ajudar na elucidação do provável mecanismo é o cálculo químicoquântico que, a partir das cargas atômicas derivadas do potencial eletrostático, dá uma estimativa do provável átomo envolvido no processo de oxidação e/ou redução. Os métodos que utilizam orbitais moleculares têm sido utilizados nos cálculos de diversos parâmetros de interesse químico e farmacológico e dentre estes parâmetros dois são muito utilizados em estudos das relações estrutura-atividade: energia do HOMO ("Highest Occupied Molecular Orbital" orbital molecular ocupado de mais alta energia) e energia do LUMO ("Lowest Unoccupied Molecular Orbital" - orbital molecular desocupado de menor energia) ${ }^{9}$.

Deste modo, este trabalho teve por objetivo estudar as reações de oxidação da amitriptilina sobre eletrodo compósito grafite (60\%)/ poliuretana $(40 \%)$, a fim de elucidar o provável mecanismo de oxidação com o auxílio dos cálculos quânticos e avaliar a utilização do eletrodo compósito para futuras aplicações analíticas.

\section{PARTE EXPERIMENTAL}

\section{Reagentes e soluções}

Todos os reagentes utilizados nos experimentos foram de pureza analítica e usados sem purificação prévia. A água utilizada para a preparação das soluções foi purificada em sistema Milli-Q da Millipore. A solução tampão Britton Robinson (BR) $0,1 \mathrm{~mol} \mathrm{~L}^{-1}$ foi preparada a partir dos ácidos acético, fosfórico e bórico e do perclorato de sódio.

$\mathrm{O}$ valor de $\mathrm{pH}$ desejado em cada experimento foi ajustado pela adição de uma solução de hidróxido de sódio $1,0 \mathrm{~mol} \mathrm{~L}^{-1}$.

A solução estoque de amitriptilina $3,10 \times 10^{-3} \mathrm{~mol} \mathrm{~L}^{-1}$ foi preparada em meio de etanol, para a total solubilização do composto e conservada sob proteção de luz.

\section{Instrumentação}

A técnica de voltametria cíclica foi empregada para o estudo preliminar da oxidação da amitriptilina. De início, os experimentos consistiram em testes com diferentes eletrodos, no intervalo de potencial adequado a cada um, com o intuito de avaliar a resposta voltamétrica. Com o eletrodo compósito foram variados os seguintes parâmetros: pH, velocidade de varredura e concentração. Nestes experimentos, os voltamogramas cíclicos foram registrados no intervalo de 250 a $1100 \mathrm{mV} v s$. eletrodo de prata cloreto de prata $\left(\mathrm{E}_{\mathrm{Ag} / \mathrm{AgCl}(\mathrm{s})}\right)$, em um analisador polarográfico EG\&G Princeton Applied Research modelo 174 A com o uso do software AVOLM.

Vários eletrodos de trabalho foram utilizados, dentre eles os de ouro, platina e carbono vítreo, com o objetivo de comparar a resposta analítica dos compostos estudados com o eletrodo de GPU.

O eletrodo de GPU foi construído utilizando-se tarugos do compósito de 1,0 cm de comprimento e diâmetro de 3,0 mm, conectados a um fio de cobre por meio de cola de prata coloidal. $\mathrm{O}$ conjunto foi, então, inserido em um tubo de vidro de $0,4 \mathrm{~cm}$ de diâmetro e 7,0 cm de comprimento. A extremidade contrária ao compósito foi selada com um tarugo de Teflon ${ }^{\circledR}$. Este artifício permitiu que a resina de poliéster fosse adicionada e preenchesse devidamente o interior do tubo. Após o processo de cura da resina, que durou 24 h, o eletrodo pôde ser facilmente descolado do interior do tubo de vidro. $\mathrm{O}$ excesso de resina foi removido com uma lixa de granulação 600 para expor o compósito. O polimento foi feito em feltro umedecido com água, pois a utilização de suspensão de alumina provocava a adesão de partículas na superfície do eletrodo, tornando demorada a etapa de polimento.

Experimentos de eletrólise a potencial controlado foram realizados para a determinação do número de elétrons envolvidos na reação de oxidação da amitriptilina. A instrumentação utilizada foi a mesma mencionada acima para a voltametria cíclica. $\mathrm{Na}$ ausência de uma placa do compósito com área suficientemente grande para a realização dos experimentos de eletrólise a potencial controlado, optou-se por utilizar uma placa de platina de área igual a $4,2 \mathrm{~cm}^{2}$.

O método semi-empírico PM3 ("Parametric Model" 3) ${ }^{10}$, encontrado no programa computacional AMPAC $6.5^{11}$, foi utilizado na otimização da geometria molecular da amitriptilina, bem como no cálculo das cargas atômicas derivadas do potencial eletrostático. A representação gráfica do HOMO (orbital molecular de mais alta energia ocupado) foi obtida utilizando o programa Spartan 5.0 ${ }^{12}$.

\section{RESULTADOS E DISCUSSÃO}

\section{Estudo voltamétrico}

\section{Testes de eletrodos}

O estudo preliminar da reação de oxidação da amitriptilina consistiu em testes para avaliar o perfil voltamétrico com diferentes eletrodos e compará-los com o eletrodo de GPU, verificando assim a possibilidade de aplicação deste eletrodo nos estudos voltamétricos, como também no desenvolvimento de metodologia analítica. A Figura 2 mostra os voltamogramas cíclicos registrados com os seguintes eletrodos de trabalho: (I) platina $(\phi=0,5 \mathrm{~mm})$; (II) ouro $(\phi=1,0 \mathrm{~mm})$; (III) carbono vítreo $(\phi=5,0 \mathrm{~mm})$ e (IV) GPU $(\phi=3,0 \mathrm{~mm})$.

É possível observar que a oxidação da amitriptilina se inicia próximo de $750 \mathrm{mV}$ para todos os eletrodos. No entanto, verificase que para os eletrodos de ouro e platina a oxidação eletroquímica ocorre na mesma região de potenciais onde há a formação de seus respectivos óxidos, resultando na dificuldade de análise do comportamento oxidativo do composto. Quanto aos eletrodos de carbono vítreo e de GPU, foram obtidas respostas voltamétricas bem mais definidas na região de potenciais entre 750 a 1200 mV. Com base nesses resultados, o eletrodo compósito foi escolhido como eletrodo de trabalho, por conciliar o melhor perfil voltamétrico e maior sensibilidade analítica.

\section{Perfil voltamétrico da amitriptilina}

Com a escolha do eletrodo de trabalho, o perfil voltamétrico da amitriptilina foi registrado por meio de sucessivas ciclagens de potencial, como mostrado na Figura 3(I). No primeiro ciclo, na região de potenciais entre 750 a $1200 \mathrm{mV}$, pode-se observar 2 picos, em 830 e $1025 \mathrm{mV}$, respectivamente. O primeiro pico é atribuído à oxidação irreversível da amitriptilina ${ }^{5}$ e o segundo, não relatado na literatura, pode estar relacionado a um processo de adsorção forte de reagente, ou seja, um pós-pico de adsorção ${ }^{13}$. $\mathrm{O}$ decréscimo bem pronunciado na corrente após a segunda ciclagem de potencial é um indicativo de bloqueio da superfície eletródica, por adsorção de produtos de oxidação da amitriptilina.

A Figura 3(II) mostra o efeito de vários tempos de pré-concentração na corrente de pico referente à oxidação da amitriptilina, podendo-se observar que ao aplicar diferentes tempos de pré-concentração ocorre um aumento na resposta voltamétrica, o que pode ser explicado pela acumulação por adsorção do composto na superfície do eletrodo.

Deste modo, pelos perfis voltamétricos exibidos constata-se 
que a amitriptilina se oxida irreversivelmente, com adsorção de reagente e produtos de oxidação.
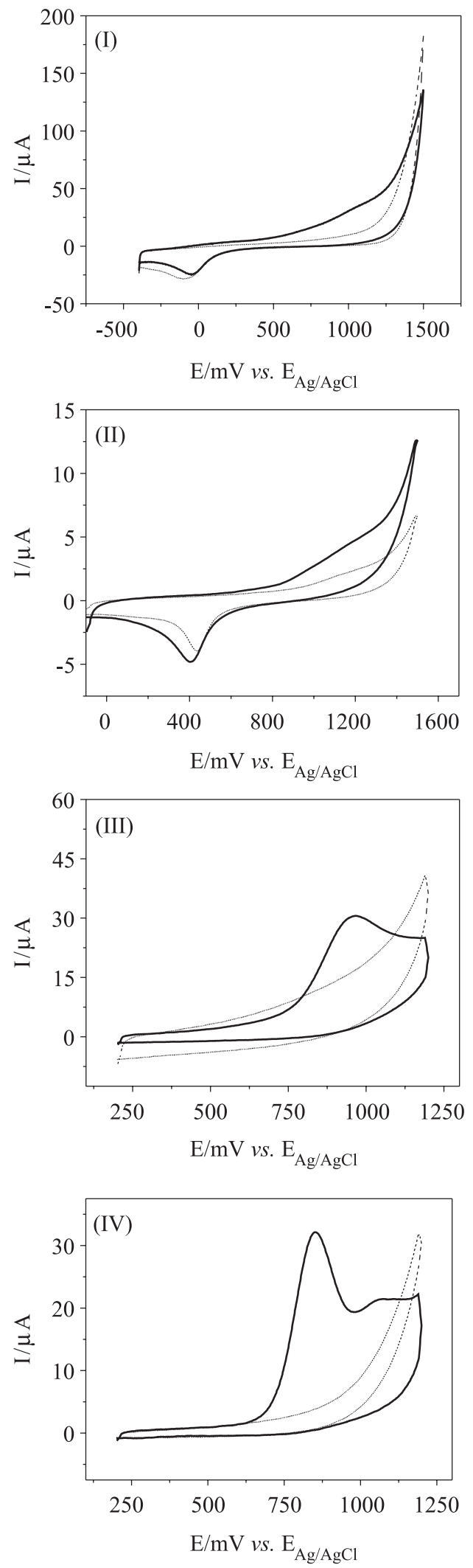

Figura 2. Voltamogramas cíclicos: (---) tampão BR 0,1 mol L-1 $(p H$ 7) $e(-)$ solução de amitriptilina $2,22 \times 10^{-4} \mathrm{~mol} \mathrm{~L}^{-1}$ em tampão BR 0,1 $\mathrm{mol} \mathrm{L}^{-1}$ (pH 7). Eletrodos de trabalho: (I) platina; (II) ouro; (III) carbono vítreo e (IV) GPU $v=100 \mathrm{mV} \mathrm{s}^{-1}$
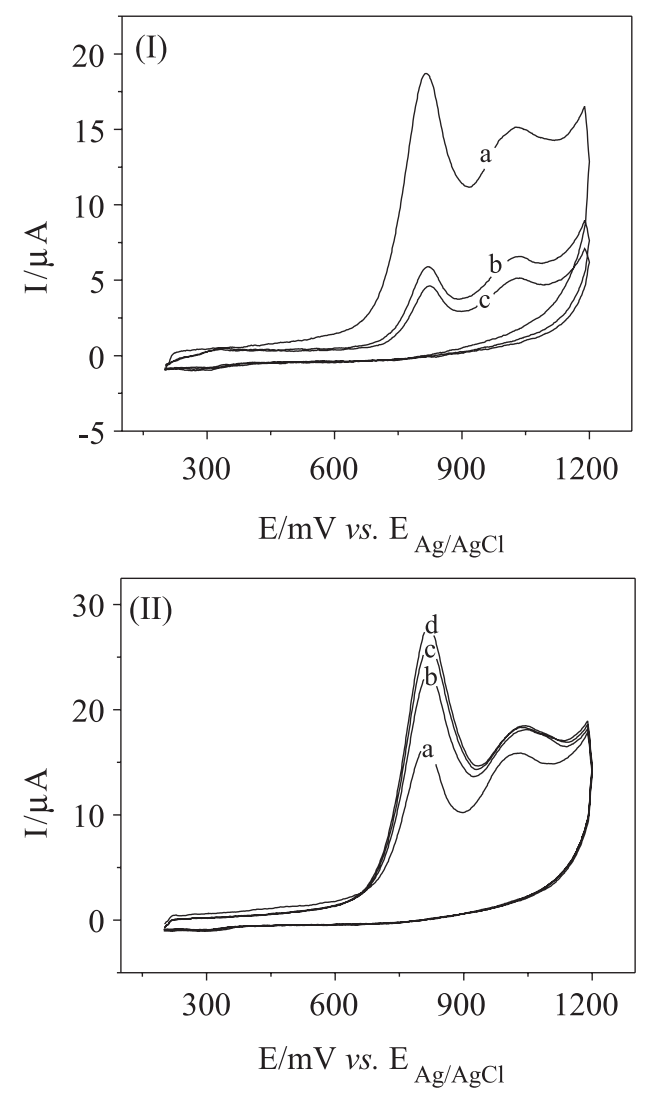

Figura 3. Voltamogramas cíclicos, mostrando: (I) várias ciclagens de potencial, (a) $1^{\circ},(b) 2^{\circ} e$ (c) $3^{\circ}$ ciclos e (II) vários tempos de pré-concentração, (a) $0 \mathrm{~s}$, (b) $30 \mathrm{~s}$, (c) $60 \mathrm{se} \mathrm{e} \mathrm{(d)} 90 \mathrm{~s}$, com potencial de pré-concentração em $250 \mathrm{mV}$. Solução de amitriptilina $2,22 \times 10^{-4} \mathrm{~mol} \mathrm{~L}^{-1}$ em tampão BR 0,1 $\mathrm{mol} \mathrm{L} \mathrm{L}^{-1}$ (pH 7). Eletrodo de trabalho: $G P U(\phi=3,0 \mathrm{~mm}) \cdot v=100 \mathrm{mV} \mathrm{s}^{-1}$

Variação da velocidade de varredura de potenciais

$\mathrm{O}$ experimento de variação da velocidade de varredura foi realizado no intervalo de 10 a $500 \mathrm{mV} \mathrm{s}^{-1}$, com o objetivo de avaliar o grau de reversibilidade e a natureza do transporte do material eletroativo para a superfície do eletrodo. A Figura 4 apresenta os voltamogramas cíclicos de uma solução de amitriptilina 2,22 x 10 mol L-1 em tampão BR $0,1 \mathrm{~mol} \mathrm{~L}^{-1}(\mathrm{pH} 7)$ em diferentes velocidades de varredura.

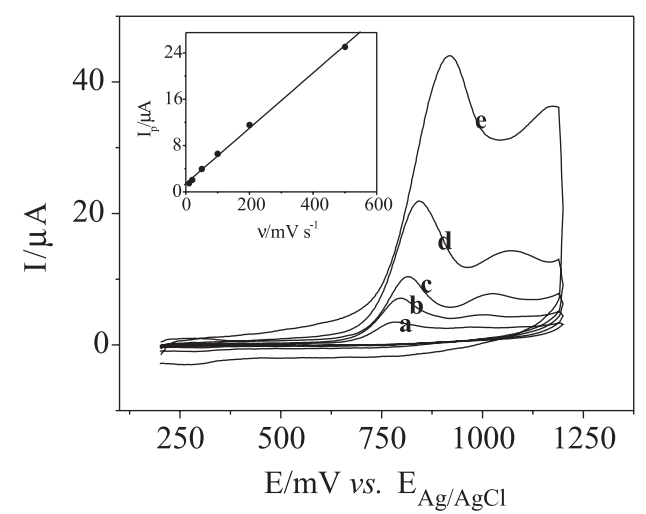

Figura 4. Voltamogramas cíclicos de uma solução de amitriptilina $2,22 \times 10^{-4}$ mol $L^{-1}$ em tampão BR 0,1 mol L-1 (pH 7). Velocidades: (a) 20; (b) 50; (c) 100; (d) 200 e (e) $500 \mathrm{mV} \mathrm{s}^{-1}$. Eletrodo de trabalho: $\mathrm{GPU}(\phi=3,0 \mathrm{~mm})$. Inserido $I_{p}$ vs. $v(R=0,9989)$ 
No intervalo de velocidade estudado, verificou-se que a amitriptilina se oxida irreversivelmente pela ausência de pico na varredura reversa de potencial e, também, pelo deslocamento dos potenciais de pico com o aumento da velocidade de varredura. A linearidade do gráfico $I_{p} v s$. v (inserido na Figura 4) confirma a ocorrência do processo de adsorção de reagente na superfície do eletrodo. Pelo critério de irreversibilidade $\left|\mathrm{E}_{\mathrm{p}}-\mathrm{E}_{\mathrm{p} / 2}=48 / \mathrm{n} \alpha\right|$, foi possível calcular o valor de n $\alpha$ como sendo igual a 0,77 , com $n$ referindo-se ao número de elétrons envolvidos na reação e $\alpha$ ao coeficiente de transferência de carga, que constitui uma medida da simetria da barreira de energia de ativação, aproximando-se de 0,5 para um condutor metálico e para um processo reversível simples de transferência de elétron ${ }^{14}$.

\section{Eletrólise a potencial controlado}

A técnica de eletrólise a potencial controlado foi utilizada para determinar o número de elétrons envolvidos na oxidação da amitriptilina e, conseqüentemente, calcular o valor do coeficiente de transferência de carga $(\alpha)$. Os experimentos foram realizados à temperatura ambiente, em um potencial mais positivo $(\mathrm{E}=1000 \mathrm{mV})$ que o potencial de pico da amitriptilina $\left(\mathrm{E}_{\mathrm{p}}=830 \mathrm{mV}\right)$ determinado por voltametria cíclica. Após $1200 \mathrm{~s}$ de eletrólise, a carga resultante $(\mathrm{Q}=0,080 \mathrm{C})$ ficou bastante próxima da carga $(\mathrm{Q}=0,091 \mathrm{C})$ necessária para oxidar $0,944 \times 10^{-6}$ mols de amitriptilina em um processo envolvendo a transferência de 1 elétron. Assim, com o valor de n $\alpha$ conhecido $(0,77)$, o coeficiente de transferência de carga $(\alpha)$ pôde ser calculado como sendo igual a 0,77 .

\section{Variação do $\mathrm{pH}$}

$\mathrm{O}$ estudo da variação do $\mathrm{pH}$ da solução foi realizado no intervalo de 4 a 12, com o objetivo de avaliar o número de prótons envolvidos na reação de oxidação da amitriptilina. A variação de $\mathrm{pH}$ também permitiu constatar em que valor ocorre maior resposta de corrente para fins analíticos. A Figura 5 mostra os voltamogramas cíclicos no intervalo de $\mathrm{pH}$ de 4 a 12 .

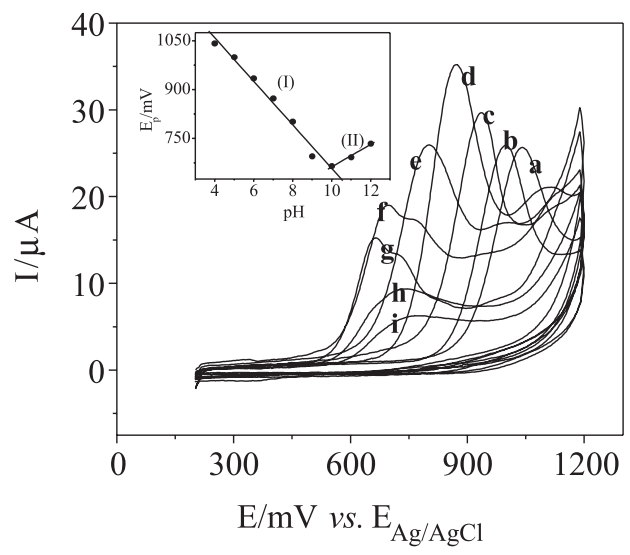

Figura 5. Voltamogramas cíclicos de uma solução de amitriptilina 2,22 $\times 10^{-4}$ mol L-1 em tampão BR 0,1 mol L-1. Valores de $\mathrm{pH}$ : (a) 4; (b) 5; (c) 6; (d) 7; (e) 8; (f) 9; (g) 10 (h) 11 e (i) 12. Eletrodo de trabalho: $G P U(\phi=3,0 \mathrm{~mm}) . v=$ $100 \mathrm{mV} \mathrm{s}^{-1}$. Inserido: $E_{p}$ vs. $\mathrm{pH}$ (Intervalo I, $R=0,9933$ e $\theta=66,93 \mathrm{mV} / \mathrm{pH}$. Intervalo II, $R=0,9922$ e $\theta=34,50 \mathrm{mV} / \mathrm{pH}$ )

Ao analisar a Figura 5, verifica-se que os potenciais de pico sofreram um deslocamento para valores menos positivos com o aumento do $\mathrm{pH}$, indicando que o mecanismo da reação eletroquímica é dependente do $\mathrm{pH}$ do meio. No intervalo de $\mathrm{pH}$ estudado, duas inclinações foram obtidas: a primeira $\left(\theta_{\mathrm{I}}=66,93 \mathrm{mV} / \mathrm{pH}\right)$ entre $\mathrm{pH} 4$ e 10, correspondendo à liberação de 1 elétron e 1 próton por molécula oxidada e a segunda $\left(\theta_{\mathrm{II}}=34,50 \mathrm{mV} / \mathrm{pH}\right)$, entre $\mathrm{pH}$ 10 e 12 , referente à saída de 2 elétrons e 1 próton.

$\mathrm{O}$ pKa da dissociação do sal de amônio-amina pôde ser estimado na intersecção das retas (I) e (II). O valor obtido (pKa 9,9) é próximo do encontrado na literatura $(\mathrm{pKa} 9,4)^{15}$.

Ao analisar a Figura 5 verifica-se que a partir do $\mathrm{pH} 9$ surgem ondas compostas, o que é um indicativo de mudança de mecanismo da reação, já que neste valor de $\mathrm{pH}$ ocorre a desprotonação do sal de amônio gerando a amina correspondente. Assim, o par de elétrons livres do nitrogênio também passa a ser uma região com densidade de carga negativa e, portanto, passível de sofrer oxidação.

Para fins analíticos, constatou-se que em $\mathrm{pH} 7$ se obteve maior resposta de corrente para a oxidação da amitriptilina.

\section{Variação da concentração}

Os experimentos de variação da concentração foram realizados a partir de uma solução estoque $3,10 \times 10^{-3} \mathrm{~mol} \mathrm{~L}^{-1}$, adicionando-se $100 \mu \mathrm{L}$ desta solução em $10 \mathrm{~mL}$ de tampão BR. As alíquotas adicionadas foram em número de seis, correspondendo a concentrações no intervalo de 1,49 a $8,74 \times 10^{-5} \mathrm{~mol} \mathrm{~L}^{-1}$. A Figura 6 mostra os voltamogramas cíclicos registrados neste intervalo de concentração.

A curva $\mathrm{I}_{\mathrm{p}}$ vs. $\mathrm{C}_{\mathrm{AM}}$ (inserida na Figura 6) apresentou boa linearidade $\left(\mathrm{R}^{\mathrm{p}}=0,9981\right)$ no intervalo de concentração estudado, mostrando que o eletrodo de GPU pode ser utilizado para a determinação analítica do composto aplicando técnicas de pulso.

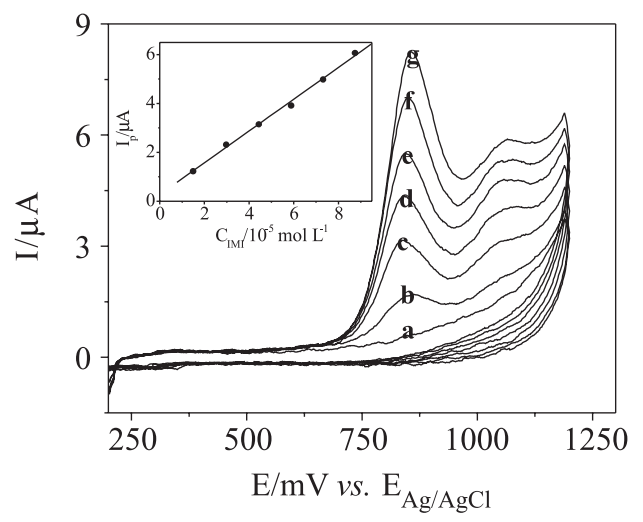

Figura 6. Voltamogramas cíclicos, variando-se a concentração de amitriptilina adicionada: (a) branco; (b) 1,49; (c) 2,97; (d) 4,43; (e) 5,88;

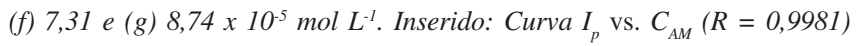
Eletrodo de trabalho: $G P U(\phi=3,0 \mathrm{~mm}) . v=50 \mathrm{mV} \mathrm{s}$

\section{Estudo químico-quântico}

Pelo emprego da voltametria cíclica, pode-se constatar que a reação de oxidação da amitriptilina corresponde a um processo irreversível controlado pela adsorção de reagente e produto, ocorrendo a liberação de 1 elétron e 1 próton. No entanto, para melhor entendimento do processo eletroquímico, foi realizado um estudo químico-quântico com o objetivo de determinar o provável sítio de oxidação da molécula.

A primeira etapa do estudo consistiu na otimização da geometria da molécula no estado fundamental (protonada e com carga +1 ) e após a oxidação (protonada e com carga +2 ). A seguir, foi realizado o cálculo do orbital molecular de fronteira (HOMO) da molécula de amitriptilina no estado fundamental. O orbital molecular de mais alta energia ocupado (HOMO) é representado graficamente na Figura 7. 


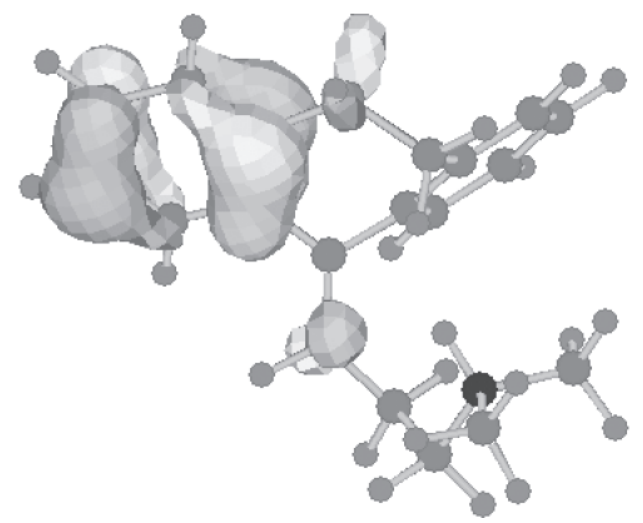

Figura 7. Representação gráfica do HOMO para a molécula de amitriptilina no estado fundamental (protonada e com carga +1 )

Os orbitais moleculares HOMO e LUMO têm sido muito utilizados em estudos de reatividade química, pois indicam os possíveis sítios reativos em reações de oxidação e redução. Uma análise do orbital HOMO fornece resultados sobre a tendência de um composto em formar um cátion, pois é deste orbital que o elétron seria retirado em uma reação de oxidação. Já o orbital LUMO fornece indícios sobre os sítios de redução de uma molécula, pois este orbital é o responsável pela entrada de elétrons na molécula. Como neste trabalho a reação envolvendo a amitriptilina é uma reação de oxidação, somente o orbital HOMO (orbital molecular de onde sairá o elétron referente à oxidação da amitriptilina) será analisado. A partir da Figura 7 observa-se que os orbitais dos átomos C8, C9, C10, C12, C13, C14, C15 e C16 são os que apresentam maior contribuição para a formação do orbital molecular HOMO, sendo, portanto, estas regiões de extrema importância no processo de oxidação da molécula.

Com o intuito de confirmar as evidências constatadas a partir da análise do orbital molecular HOMO, foi efetuado o cálculo das cargas atômicas derivadas do potencial eletrostático ${ }^{16}$, com a molécula no estado fundamental e após a oxidação. Este cálculo permitiu avaliar a mudança de densidade eletrônica antes e após a oxidação da molécula. Os valores das cargas atômicas calculadas estão indicados na Tabela 1.

Ao analisar a Tabela 1, verifica-se que a remoção de um elétron leva a mudanças na densidade eletrônica da molécula, porém a variação mais significativa ocorre no átomo C16 (aproximadamente, carga $=-0,3$ na molécula no estado fundamental e carga $=$ 0,0 na molécula após a oxidação). Os outros possíveis sítios de oxidação, segundo a Figura 7 (átomos C9, C10, C12, C13, C14 e C15), estão localizados em um anel aromático e, desta forma, ocorre uma deslocalização das cargas nestes sítios, o que explica pequenas variações encontradas nos valores de cargas. Portanto, o átomo C16 é, segundo os cálculos de densidade de carga, o principal sítio de oxidação da molécula de amitriptilina. A Figura 1 mostra a estrutura da molécula com os átomos numerados de acordo com a Tabela 1. A perda de um próton também foi analisada, e duas possibilidades foram estudadas: perda de um próton no átomo $\mathrm{C} 17$ ou no átomo $\mathrm{C} 18$. De acordo com as cargas atômicas apresentadas na Tabela 1, a maior densidade de carga negativa, referente à perda de um $\mathrm{H}^{+}$, ocorre no átomo $\mathrm{C} 17$, sugerindo que este seria o provável sítio de saída do próton.

O provável mecanismo de oxidação da amitriptilina (Figura 8) foi sugerido de acordo com os resultados eletroquímicos e os cálculos químico-quânticos. Pelos resultados eletroquímicos, o processo de oxidação da amitriptilina sobre GPU ocorre com a perda de 1 elétron e 1 próton. De acordo com a Tabela 1, o átomo C16 apresentou a maior variação de densidade de carga na molécula e,
Tabela 1. Cálculo das cargas atômicas derivadas do potencial eletrostático para a molécula de amitriptilina, no estado fundamental e após a oxidação

\begin{tabular}{ccccc}
\hline Átomos & $\begin{array}{c}\text { Estado } \\
\text { fundamental }\end{array}$ & $\begin{array}{c}\text { Após } \\
\text { oxidação }\end{array}$ & $\begin{array}{c}\text { Sem H } \\
\text { no C17 }\end{array}$ & $\begin{array}{c}\text { Sem H } \\
\text { no C18 }\end{array}$ \\
\hline 1 C & $-0,26$ & $-0,32$ & $-0,23$ & $-0,26$ \\
2 C & $-0,07$ & 0,09 & $-0,09$ & $-0,08$ \\
3 C & 0,09 & 0,19 & 0,08 & 0,09 \\
4 C & $-0,19$ & $-0,06$ & $-0,19$ & $-0,19$ \\
5 C & 0,0 & 0,28 & $-0,02$ & $-0,01$ \\
6 C & $-0,13$ & $-0,31$ & $-0,15$ & $-0,15$ \\
7 C & $-0,21$ & $-0,22$ & $-0,24$ & $-0,22$ \\
8 C & $-0,09$ & $-0,16$ & $-0,22$ & $-0,08$ \\
9 C & 0,0 & $-0,08$ & 0,11 & $-0,03$ \\
10 C & 0,11 & 0,19 & 0,06 & 0,10 \\
$11 \mathrm{C}$ & $-0,08$ & $-0,21$ & 0,0 & $-0,04$ \\
$12 \mathrm{C}$ & $-0,25$ & $-0,15$ & $-0,25$ & $-0,21$ \\
13 C & $-0,13$ & $-0,11$ & $-0,14$ & $-0,12$ \\
$14 \mathrm{C}$ & $-0,16$ & $-0,09$ & $-0,15$ & $-0,16$ \\
$15 \mathrm{C}$ & $-0,19$ & $-0,33$ & $-0,22$ & $-0,17$ \\
$16 \mathrm{C}$ & $-0,29$ & $-0,03$ & $-0,25$ & $-0,29$ \\
17 C & 0,02 & $-0,03$ & $-0,23$ & 0,04 \\
18 C & $-0,41$ & $-0,55$ & $-0,29$ & $-0,54$ \\
19 N & 0,73 & 0,76 & 0,79 & 0,89 \\
20 C & $-0,38$ & $-0,47$ & $-0,47$ & $-0,45$ \\
21 C & $-0,51$ & $-0,54$ & $-0,45$ & $-0,48$ \\
\hline
\end{tabular}

desta forma, as especulações mecanísticas foram baseadas nesta região da molécula. Após a perda do elétron, que ocorre no átomo C16, há a saída de um próton no átomo C17 devido à maior densidade de carga negativa neste átomo, após a oxidação da molécula. Em seguida, há o rearranjo de cargas por meio do compartilhamento da carga negativa entre os átomos $\mathrm{C} 16$ e C17, gerando um radical cátion (no C11) como produto da reação de oxidação da amitriptilina nas condições estudadas.

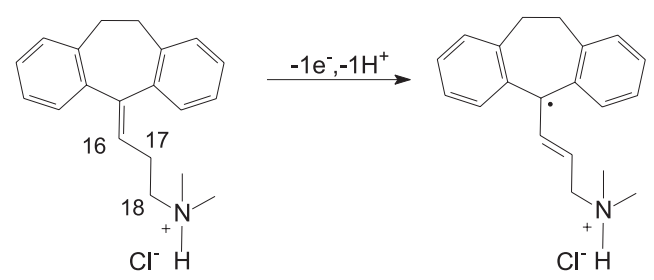

Figura 8. Possível mecanismo de oxidação da amitriptilina sobre GPU

\section{CONCLUSÕES}

O estudo voltamétrico da reação de oxidação do antidepressivo tricíclico amitriptilina com o eletrodo de GPU mostrou que o composto se oxida irreversivelmente com a transferência de 1 elétron e 1 próton, sendo a reação controlada pela adsorção de reagente e produto. Estudos para o desenvolvimento de metodologia para a determinação analítica da amitriptilina, por voltametria de onda quadrada, estão em andamento com a investigação das demais condições experimentais, como a freqüência de aplicação dos pulsos de potencial, a amplitude de pulso e o incremento de varredura. A representação do HOMO mostra uma grande contribuição dos átomos C8, C9, C10, C12, C13, C14, C15 e C16 para a formação deste orbital, indicando que estes átomos seriam os possíveis sítios de oxidação da molécula. Os cálculos das cargas dos átomos da molécula no 
estado fundamental e após a oxidação evidenciaram uma maior variação da densidade de carga no átomo $\mathrm{C} 16$, indicando que este átomo é o principal sítio de oxidação da molécula. Além disso, um possível mecanismo para a oxidação da amitriptilina foi sugerido. Experimentos de eletrólise exaustiva estão sendo realizados com a finalidade de se identificar os prováveis produtos de oxidação, para dar maior embasamento ao mecanismo proposto.

\section{AGRADECIMENTOS}

Os autores agradecem à FAPESP (Proc. no 01/14629-0 e 01/ 06029-3) e ao CNPq.

\section{REFERÊNCIAS}

1. Ban, T. A.; Prog. Neuro-Psychopharmacol. Biol. Psychiat. 2001, 25, 709.

2. Sussman, N.; Stahl, S.; The Amer. J. Med. 1996, 101, 26S.
3. Bishop, E.; Hussein, W.; Analyst 1984, 109, 73.

4. Turk, D. J.; McClintock, S. A.; Purdy, W. C.; Anal. Lett. 1985, 18, 2605.

5. Biryol, I.; Uslu, B.; Küçükyavuz, Z.; J. Pharm. Biol. Anal. 1996, 15, 371.

6. Ivandini, T. A.; Sarada, B. V.; Terashima, C.; Rao, T. N.; Tryk, D. A.; Ishiguro, H.; Kubota, Y.; Fujishima, A.; J. Electroanal. Chem. 2002, 521 , 117.

7. Pereira, C. A.; Santos, S. A.; Kubota, L. T.; Quim. Nova 2002, 25, 1012.

8. Mendes, R. K.; Claro-Neto, S.; Cavalheiro, E. T. G.; Talanta 2002, 57, 909.

9. Clare, B. W.; Theor. Chim. Acta 1994, 87, 415.

10. Stewart, J. J. P.; J. Comput.-Aided Mol. Des. 1990, 4, 1.

11. Dewar, M. J. S.; AMPAC 6.5: Program for semiempirical calculations; Universidade do Texas, Estados Unidos, 1994.

12. Hehre, W. J.; Huang, W. W.; Klunzinger, P. E.; Deppmeier, B. J.; Driessen, A. J.; Spartan 5.0: User's guide; Irvine, CA., 1996.

13. Wepschall, R. H.; Sahin. I.; Anal. Chem. 1967, 39, 1514.

14. Brett, A. M. O.; Brett, C. M. A.; Electroquímica: Princípios, Métodos e Aplicações, Oxford University Press: Coimbra, 1993.

15. http://chrom.tutms.tut.ac.jp/JINNO/DRUGDATA/55nortriptyline.html, acessada em Junho 2004

16. Bruns, R. E.; Guadagnini, P. H.; Souza, A. A.; Quim. Nova 1996, 19, 148. 